\title{
The significance of an integrated management mode of prenatal diagnosis-postnatal treatment for critical congenital heart disease in newborns
}

\author{
Xiaohui Zhang ${ }^{1}$, Shaoru He ${ }^{2}$, Yumei Liu ${ }^{1}$, Jing Zhong ${ }^{1}$, Yunxia Sun ${ }^{1}$, Manli Zheng ${ }^{1}$, Juan Gui ${ }^{1}$, \\ Ruixi Wang ${ }^{1}$, Bowen Feng ${ }^{1}$, Jianling Mo ${ }^{1}$, Minqiao Jian ${ }^{3}$, Caisheng Liu ${ }^{3}$, Yijing Liang ${ }^{1}$ \\ ${ }^{1}$ Department of NICU, Guangdong Cardiovascular Institute, Guangdong Provincial People's Hospital, Guangdong Academy of Medical Sciences, \\ Guangzhou, China; ${ }^{2}$ Department of NICU, Guangdong Provincial People's Hospital, Guangdong Academy of Medical Sciences, Guangdong \\ Cardiovascular Institute, Guangzhou, China; ${ }^{3}$ Department of NICU, Guangdong Provincial People's Hospital, School of Medicine, South China \\ University of Technology, Guangzhou, China \\ Contributions: (I) Conception and design: X Zhang, S He, Y Liu; (II) Administrative support: C Liu, Y Liang; (III) Provision of study materials or \\ patients: B Feng, J Mo, M Jian; (IV) Collection and assembly of data: R Wang, J Gui; (V) Data analysis and interpretation: J Zhong, Y Sun, M Zheng; \\ (VI) Manuscript writing: All authors; (VII) Final approval of manuscript: All authors. \\ Correspondence to: Shaoru He, MD. Department of NICU, Guangdong Cardiovascular Institute, Guangdong Provincial People's Hospital, \\ Guangdong Academy of Medical Sciences, 106 Zhongshan 2nd Road, Guangzhou 510080, China. Email: Heshaoru2020@yeah.net.
}

Background: Congenital heart disease (CHD) is the most common congenital defect in human beings. The purpose of this article is to investigate impact of an integrated management mode of 'prenatal diagnosispostnatal treatment' on birth, surgery, prognosis and complications associated with critical CHD (CCHD) in newborns.

Methods: A retrospective analysis of the medical records of newborns diagnosed with CCHD were divided into two groups: prenatal diagnosis and postnatal diagnosis. The demographics, clinical characteristics, surgical status, prognosis and complications of the two groups were compared and the differences identified.

Results: Among the 290 newborns with CCHD, 97 (33.4\%) were prenatally diagnosed and 193 (66.6\%) were postnatally diagnosed. Newborns in the prenatal diagnostic group were hospitalized immediately after birth, whereas the median age of admission was 6.00 (3.00-12.00) days in postnatal diagnostic group, $\mathrm{P}=0.000$. In terms of postnatal symptoms and signs, the incidence of anhelation, cyanosis and cardiac murmur was higher in the postnatal diagnostic group. The rates of preoperative intubation, postoperative open chest exploration and treatment abandonment were higher in the postnatal diagnostic group. The postnatal diagnostic group was more prone to postoperative complications, such as pneumonia and hypoxicischemic brain damage. The preoperative mortality [0 (0.0\%) vs. $12(6.2 \%), \mathrm{P}=0.028]$ in the prenatal diagnostic group was lower than that in the postnatal diagnostic group. And the one-year survival rate of the prenatal diagnostic group was higher (log-rank test $\mathrm{P}=0.034)$.

Conclusions: The integrated management mode of prenatal diagnosis-postnatal treatment can improve postnatal symptoms, reduces complications, reduces preoperative mortality and increases one-year survival rates in newborns with CCHD.

Keywords: Fetus; critical congenital heart disease (critical CHD); prenatal diagnosis; postnatal treatment; integrated management

Submitted Oct 29, 2020. Accepted for publication Jan 18, 2021.

doi: $10.21037 / \mathrm{cdt}-20-892$

View this article at: http://dx.doi.org/10.21037/cdt-20-892 


\section{Introduction}

Congenital heart disease (CHD) is the most common congenital defect in human beings, with an incidence of $8-12 / 1,000(1-3)$, and is one of the significant causes of infant death (4). Approximately one-third of CHD is critical CHD (CCHD) $(5,6)$. If it is not diagnosed and treated in time, most children with CCHD will die from serious complications within the first year of life. The quality of life for untreated survivors will also be seriously affected, imposing a significant medical, economic and spiritual burden on families and society (7).

After the first report of foetal echocardiography for the diagnosis of severe cardiac malformations in 1986 (8), the prenatal diagnosis of CHD became a reality. Over the past three decades, with the development of ultrasound technology, the rate of prenatal diagnosis of CCHD has gradually increased from $21 \%$ to $80 \%$ (9-14). The integrated management mode of prenatal diagnosispostnatal treatment originated in developed countries in the 1990s. It is an integrated management mode of prenatal diagnosis, prenatal consultation and postnatal collaborative treatment. This mode is highly specialized and helps to optimize medical resources and improve treatment outcomes. The mode is multidisciplinary and covers the natural course of the disease at different times during the prenatal and postnatal periods.

The specific procedures of the integrated management mode of prenatal diagnosis-postnatal treatment are as follows $(15,16)$ : (I) prenatal diagnosis: the obstetric ultrasonographer performs a detailed foetal examination of the pregnant woman who is high risk for possible CHD or whose foetus is suspected of having CHD from an external screening ultrasound of the heart. (II) Prenatal consultation: the paediatric cardiologist shall inform the pregnant woman and her family of the symptoms, treatment plan, efficacy and costs involved for the foetus after birth, as well as the technical ability and associated risks of the current CHD treatment, and suggest that she carries out relevant tests to exclude the foetal chromosome disease and other system diseases. It is up to the pregnant woman and her family to decide whether or not to continue the pregnancy. (III) Prenatal examination and delivery: the doctor shall guide the woman who has decided to continue the pregnancy on having a prenatal examination and a delivery in a medical institution with CHD treatment ability. If there is no evidence of caesarean section, it is recommended that the pregnant woman gives birth naturally, if possible.
(IV) Neonatal treatment: the echocardiography shall be checked as early as possible after birth to make a clear diagnosis. Some newborns require an additional cardiac spiral CT to confirm the diagnosis. According to the severity of the CHD, the function of heart and lungs and the general condition of the patients, neonatal experts, paediatric cardiologists and paediatric cardio surgeons work out the treatment plan together. (V) Surgical treatment: if it is difficult to correct the hypoxia or heart failure after medical treatment, intervention or surgical operation shall be performed in the neonatal period. (VI) Follow up: after discharge, the patient shall be followed up in the outpatient department, and the echocardiography is to be re-examined regularly to evaluate the structure and function of the heart.

The integrated management mode of prenatal diagnosispostnatal treatment aims to improve the postnatal state and quality of life of newborns with CCHD. However, in the published research, the effect of this mode on CCHD is still not clear. Some studies have demonstrated that prenatal diagnosis can improve the preoperative status and reduce the preoperative morbidity and mortality of patients with CCHD (17-24). However, other studies have shown that the survival benefit of a prenatal diagnosis is unclear, and even that an early diagnosis can have adverse outcomes (25-31). In view of these mixed reports, our study attempts to reevaluate the relationship between prenatal diagnosis and the clinical characteristics, surgical conditions, prognosis and complications of CCHD in newborns. We present the following article in accordance with the STROBE reporting checklist (available at: http://dx.doi.org/10.21037/cdt-20-892).

\section{Methods}

In this study, CCHD was defined as cardiac structural malformation requiring intervention within 28 days of birth, and that hypoxemia may occur at some or most times after birth (32). It mainly includes the following 11 defects (33-34): (I) the complete transposition of great arteries; (II) left ventricular hypoplasia syndrome; (III) pulmonary atresia (intact ventricular septum/ventricular septal defect); (IV) tetralogy of Fallot; (V) total anomalous pulmonary venous drainage; (VI) tricuspid atresia; (VII) persistent truncus arteriosus; (VIII) interruption of aortic arch; (IX) double outlet of right ventricle; (X) Ebstein anomaly; and (XI) single ventricular. Because the severity of the CCHD was not considered in this study, cardiac defects such as severe pulmonary artery stenosis and severe aortic stenosis were not included. 
This study retrospectively collected clinical data from 1 January 2017 to 31 December 2019 on neonates (age $<28$ days) diagnosed with CCHD at the Guangdong Provincial People's Hospital, excluding patients who had severe, life-threatening extracardiac or chromosomal abnormalities. For the prenatal diagnosis group, CCHD was detected by foetal cardiac ultrasound before birth, and there was a prenatal consultation and hospitalization immediately after birth. For the postnatal diagnosis group, CCHD was first detected by echocardiography after birth. The data of the two groups were analysed comparatively. The data collected included demographic information and preoperative, operative and postoperative data. The demographic information included age, gender, maternal age, pregnancy complications, parity, gestational week, twin or multiple pregnancies, mode of delivery, Apgar score and birth weight. The preoperative data included admission weight, admission age, postnatal symptoms and signs (anhelation, cyanosis and cardiac murmur), diagnosis time, foetal and postnatal echocardiography results, cardiac defect type, length of hospital stay, hospitalization cost, treatment modalities, prostaglandin use and the duration of mechanical ventilation. The surgical information included the type of surgery, age at time of surgery, weight at time of surgery, intraoperative blood loss, extracorporeal circulation time, aortic arch-blocking time and time of surgery. The postoperative conditions included delayed chest closure status, open chest exploration, the need for extracorporeal membrane oxygenation (ECMO), prognosis, postoperative complications and hospital mortality. The postoperative complications included respiratory failure, pneumonia, low cardiac output syndrome, multiple organ dysfunction syndrome (MODS), infections (catheter-related, septicaemia, incision infection, necrotizing enterocolitis), pneumothorax, pulmonary atelectasis, pleural effusion, diaphragmatic paralysis, venous thrombosis, arrhythmia and hypoxic-ischemic brain damage.

\section{Statistical analysis}

SPSS software version 26.0 was used for the statistical analysis. The data were reported as frequencies and percentages for categorical variables and as means and standard deviations for continuous variables. Comparisons were made using the chi-square test for categorical variables and the Wilcoxon rank sum test for continuous variables. The Kaplan-Meier survival curve and log-rank test were used to evaluate the difference in one-year survival rate between the prenatal diagnosis group and the postnatal diagnosis group. $\mathrm{P}$ values $<0.05$ were considered statistically significant.

\section{Ethical statement}

The study was conducted in accordance with the Declaration of Helsinki (as revised in 2013). The study was approved by the ethics committee of Guangdong Provincial People's Hospital (No. GDREC2018317H) and informed consent was taken from all the patients.

\section{Results}

Of the 290 CCHD newborns who met the inclusion criteria, 97 cases (33.4\%) were prenatal diagnosis and 193 cases $(66.6 \%)$ were postnatal diagnosis. There were 98 cases $(33.8 \%)$ in 2017, 103 cases $(35.5 \%)$ in 2018 and 89 cases $(30.7 \%)$ in 2019 . The specific diagnostic categories are shown in Figure 1. In the prenatal diagnostic group, complete transposition of great arteries $(41.2 \%)$ and tetralogy of Fallot (20.6\%) predominated. However, in the postnatal diagnostic group, total anomalous pulmonary venous drainage $(29.3 \%)$ and complete transposition of great arteries (21.2\%) accounted for the majority.

\section{General information}

The basic data for the patients are shown in Table 1. There were no statistically significant differences in sex, birth weight, admission weight, premature delivery rate, Apgar 1 minute score, gestational hypertension and history of influenza in early pregnancy in the prenatal diagnostic group compared to the postnatal diagnostic group. In the prenatal diagnostic group, the maternal age [31.00 (27.0035.50) vs. 29.00 (25.00-34.00), $\mathrm{P}=0.008]$, twin pregnancy rate [14 (14.4\%) vs. $12(6.2 \%), \mathrm{P}=0.021]$, caesarean section rate $[46(47.7 \%)$ vs. 63 (32.8\%), $\mathrm{P}=0.016]$ and gestational diabetes rate $[18(18.6 \%)$ vs. $15(7.9 \%), \mathrm{P}=0.007]$ was higher, the gestational age $[38.43$ (37.00-39.29) vs. 39.14 (38.00-40.00), $\mathrm{P}=0.000]$ was lower and the Apgar 5-minute score [10.00 (9.00-10.00) vs. 10.00 (10.00-10.00), $\mathrm{P}=0.004]$ was lower. The newborns in the prenatal diagnostic group were hospitalized immediately after birth, whereas the median age of admission was 6.00 (3.00-12.00) days in the postnatal diagnostic group, $\mathrm{P}=0.000$. In terms of postnatal signs and symptoms, the incidence of anhelation $(38.1 \% \mathrm{vs}$. $79.8 \%, \mathrm{P}=0.000$ ), cyanosis ( $41.2 \%$ vs. $81.9 \%, \mathrm{P}=0.000)$ and 


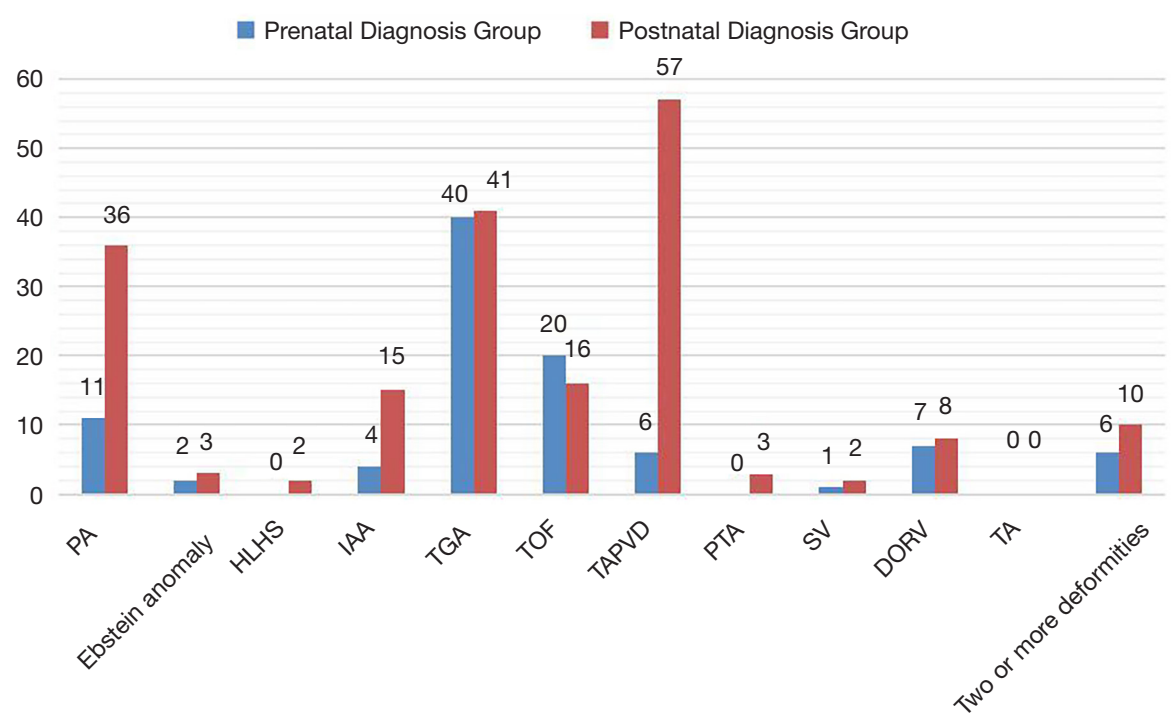

Figure 1 Anatomical types of critical congenital heart disease. PA, pulmonary atresia; HLHS, hypoplastic left heart syndrome; IAA, interrupted aortic arch; TGA, transposition of the great arteries; TOF, tetralogy of Fallot; TAPVD, total anomalous pulmonary venous drainage; PTA, persistent truncus arteriosus; SV, single ventricle; DORV, double outlet right ventricle; TA, tricuspid atresia.

Table 1 Basic information of patients

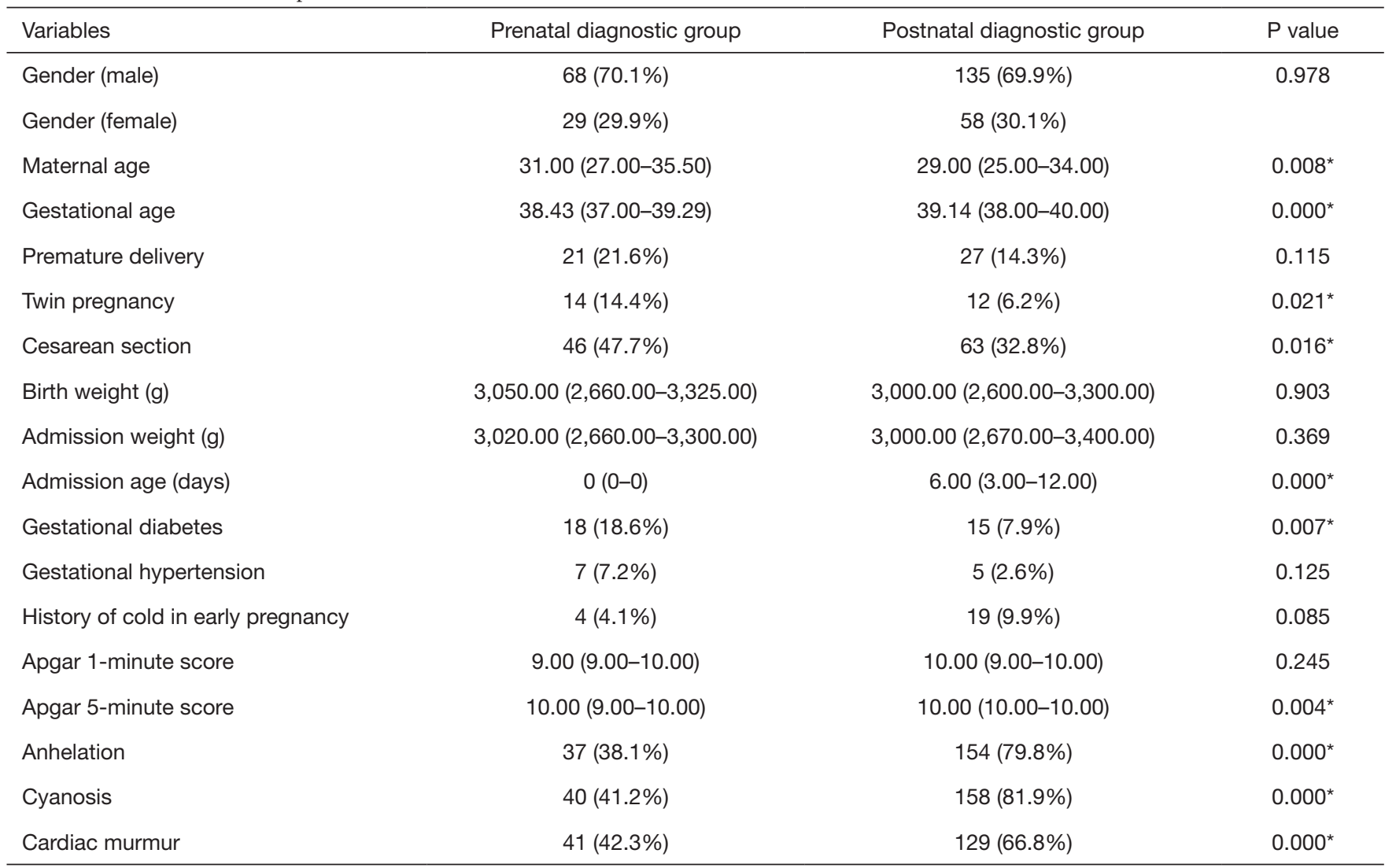

${ }^{*}, \mathrm{P}<0.05$. Data are presented as $\mathrm{n}(\%)$ or median (range). 
Table 2 Hospitalization and surgical data of the patients

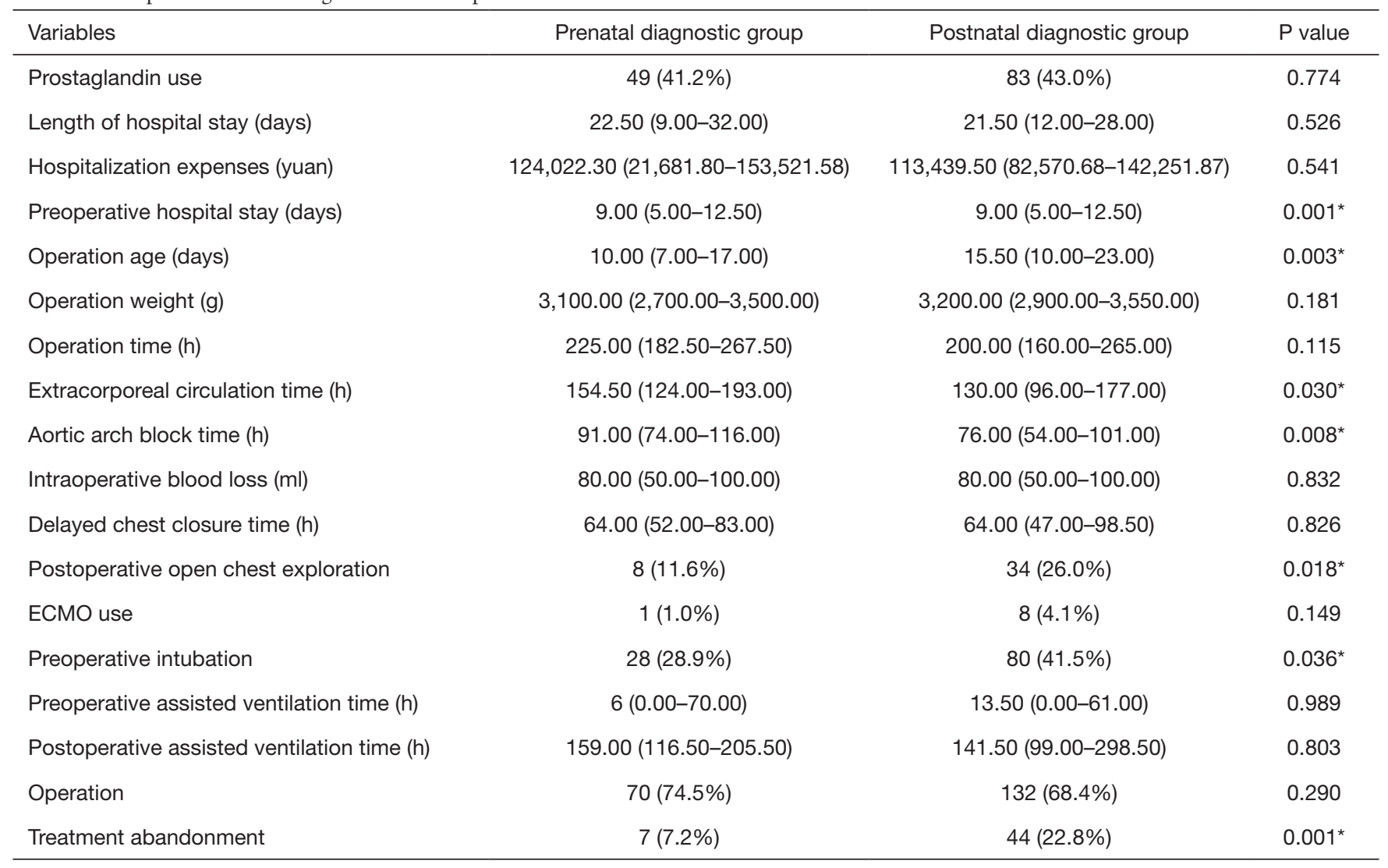

${ }^{*}, \mathrm{P}<0.05$. Data are presented as $\mathrm{n}(\%)$ or median (range). ECMO, extracorporeal membrane oxygenation.

cardiac murmur $(42.3 \%$ vs. $66.8 \%, \mathrm{P}=0.000)$ was higher in the postnatal diagnostic group.

\section{Hospitalization, surgery and post-operative conditions (Table 2)}

There were no significant differences between the two groups in postnatal prostaglandin use, length of hospital stay and hospital costs. In terms of surgery, there were no differences between the two groups in operative rate, operative weight, operative time, intraoperative blood loss, delayed chest closure time or ECMO use. In terms of assisted ventilation, there was no statistically significant difference in preoperative and postoperative assisted ventilation time between the two groups, but the preoperative intubation rate was lower in the prenatal diagnostic group [28 (28.9\%) vs. 80 (41.5\%), $\mathrm{P}=0.036$ ]. The prenatal diagnostic group had longer preoperative hospital days [9.00 (5.00 to 12.50$) v s .9 .00$ (5.00 to 12.50),
$\mathrm{P}=0.001]$, a lesser operative age $[10.00$ (7.00 to 17.00$)$ vs. 15.50 (10.00 to 23.00), $\mathrm{P}=0.003$ ] a longer operative extracorporeal circulation time [154.50 (124.00 to 193.00) vs. 130.00 (96.00 to 177.00 ), $\mathrm{P}=0.030$ ] and a longer aortic arch block time [91.00 (74.00 to 116.00) vs. 76.00 (54.00 to 101.00), $\mathrm{P}=0.008$ ]. The rate of both the postoperative open chest exploration [8 (11.6\%) vs. 34 (26.0\%), $\mathrm{P}=0.018$ ] and treatment abandonment was higher in the postnatal diagnostic group $(7.2 \%$ vs. $22.8 \%, \mathrm{P}=0.001)$.

\section{Complications and mortality (Table 3)}

Postoperative complications, such as pneumonia [26 $(28.0 \%)$ vs. $76(65.4 \%), \mathrm{P}=0.005]$ and ischemic-ischemic brain damage [0 (0.0\%) vs. 12 (7.2\%), $\mathrm{P}=0.019]$, were more common in the postnatal diagnostic group. Preoperative mortality $[0(0.0 \%)$ vs. $12(6.2 \%), \mathrm{P}=0.028]$ was lower in the prenatal diagnostic group, but postoperative and total mortality did not differ from the postnatal diagnostic group. 
Table 3 Complications and mortality

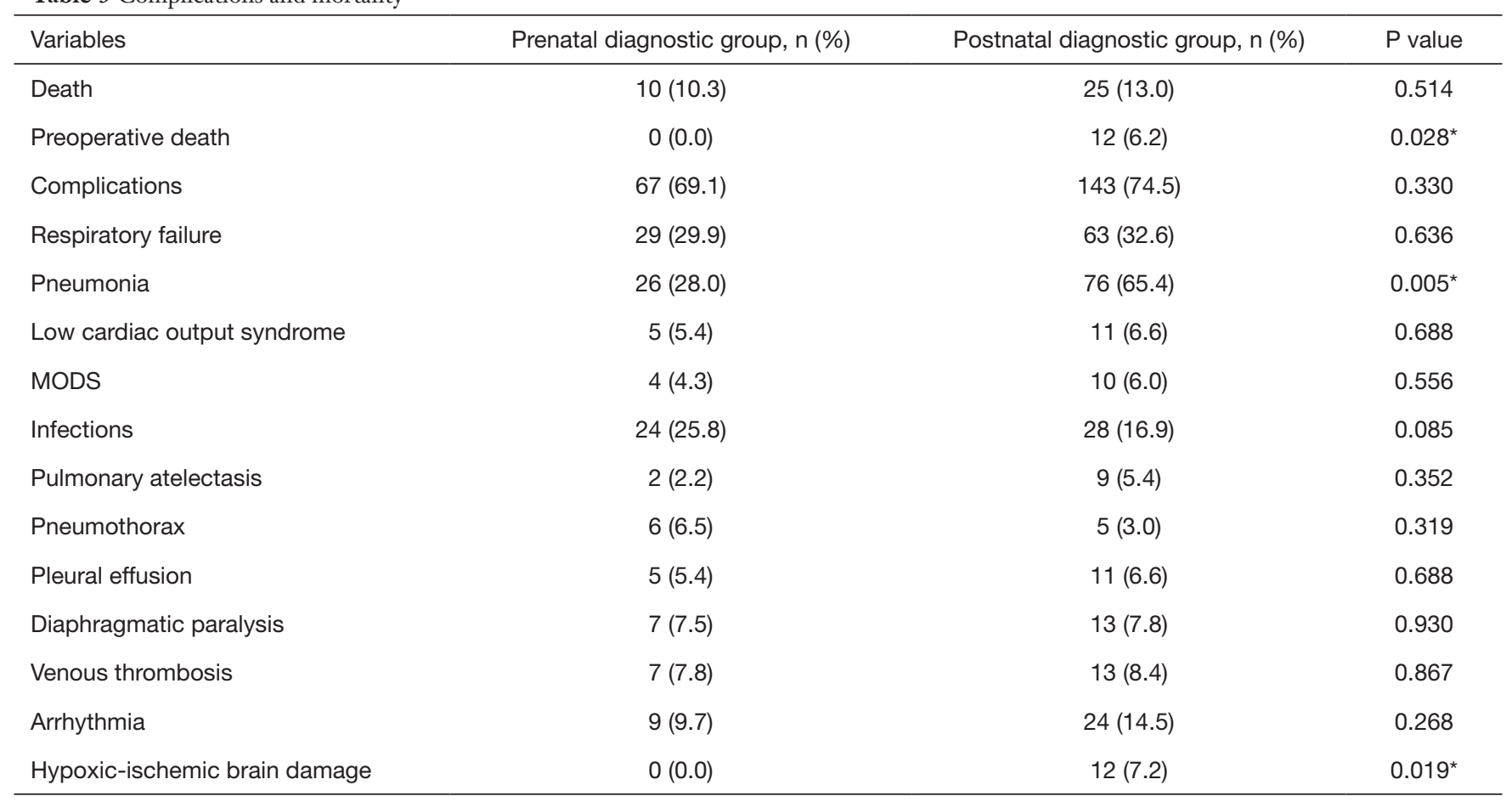

*, $\mathrm{P}<0.05$. MODS, extracorporeal membrane oxygenation.

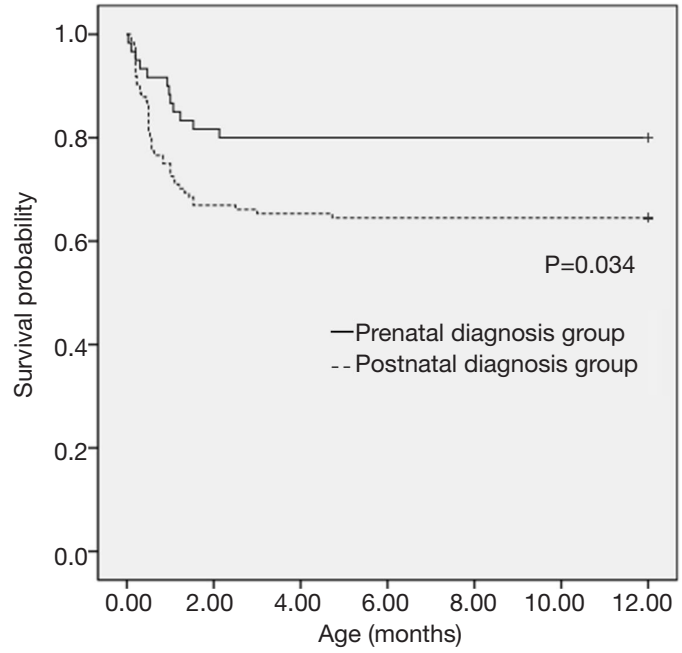

Figure 2 The Kaplan-Meier curves for the prenatal and postnatal diagnostic groups for 2017-2018.

\section{One-year survival rate}

The Kaplan-Meier curves for the two groups for 2017-2018 are shown in Figure 2. The patients in the prenatal and postnatal diagnostic groups had different survival curves (log-rank test $\mathrm{P}=0.034)$, and there was a higher one-year survival rate in the prenatal diagnostic group.

\section{Discussion}

CHD is the leading cause of morbidity and mortality in infants and young children $(35,36)$. Most CHD can be detected by secondary obstetric echocardiography in mid-pregnancy and can be confirmed by detailed foetal echocardiography. A multidisciplinary team of experts was established in conjunction with paediatrics, obstetrics, eugenics and cardiology. The experts evaluated the disease according to the ultrasonic imaging data of foetal heart disease, the pregnancy history of the mother and family history, then had a comprehensive and detailed prognosis consultation and formulated the integrated management and treatment plan for the intrauterine and neonatal period. Neonatal cardiac echocardiography is performed immediately after birth to evaluate the structural and functional changes in the neonatal heart, so that CCHD newborns can be diagnosed early and be accepting of the combined medical and surgical interventions. The aim of this study was to investigate the implications of the integrated management mode of prenatal diagnosispostnatal treatment for neonatal CCHD in the current era 
of prenatal diagnosis and prenatal consultation.

Several previous studies $(11,24,25,28,37)$ have shown that neonates with a prenatal detection of CCHD have higher rates of preterm birth, higher rates of caesarean delivery and lower birth weights, and they suggest that prenatal detection of CCHD may artificially interfere with the foetal gestational course, resulting in an earlier delivery and lower birth weight. Lower birth weight may adversely affect the postnatal status of newborns. Although this study observed smaller gestational age and higher caesarean delivery rate in the prenatal diagnostic group, there were no significant differences in the preterm birth rate, birth weight, admission weight or Apgar 1 minute score between the prenatal diagnostic group and the postnatal diagnostic group. The higher maternal age, higher rate of twin pregnancy and greater prevalence of gestational diabetes in the prenatal diagnostic group may be related to the fact that these factors were identified during pregnancy and the increased maternal screening in the prenatal diagnostic group led to the detection of CCHD in the foetus. The lower Apgar 5-minute score in the prenatal diagnostic group may be related to the complexity of the newborns' CCHD. The newborns in the prenatal diagnostic group in this study were hospitalized immediately after birth, allowing for optimal perinatal and perioperative management, involving immediate mechanical ventilation or prostaglandin therapy for newborns with catheterdependent CHD. The newborns with heart failure were treated with cardiac stimulation, diuresis and vasodilation, to combat heart failure. The median age of admission in the postnatal diagnostic group was 6.00 (3.00-12.00) days. In clinical practice, many newborns with CCHD are not diagnosed prenatally, which often leads to delayed diagnosis after birth, and most of them are in critical conditions such as severe hypoxia, acidosis and heart failure by the time they reach hospital, most of them losing precious time needed for surgery and even dying. In this study, anhelation, cyanosis and cardiac murmur were more common in the postnatal diagnostic group, indicating that delayed diagnosis had an impact on the hemodynamic stability of the newborns, and therefore their adverse clinical manifestations were more pronounced.

Oster and Verheijen et al. have shown that prenatal diagnosis reduces the incidence of postnatal oxygen deficiency, invasive respiratory support and metabolic acidosis in patients, thus avoiding hemodynamic instability, organ dysfunction and reducing surgical delays $(17,24)$. However, a single-centre study by Wright et al. of infants and young children undergoing surgery for CHD for up to five years showed that the prenatal diagnostic group had longer postoperative intensive care unit stays and longer hospital stays (38). In our study, we found that neonates in the prenatal diagnostic group had longer days of preoperative hospitalization, which may be related to the fact that newborns in the prenatal diagnostic group were hospitalized at birth and had to undergo a period of postnatal hemodynamic instability before undergoing surgery. The older the age at time of surgery, the higher the rate of preoperative intubation, and the higher rate of postoperative open chest exploration in the postnatal diagnostic group suggests that delayed diagnosis may lead to the worsening of cardiac function, the optimal time for surgery being missed and affected prognosis. Neonates in the prenatal diagnostic group had longer extracorporeal circulation time and aortic arch-block time during surgery, which may be related to the greater complexity of CCHD in neonates in the prenatal diagnostic group. Neonates in the prenatal diagnosis group had lower treatment abandonment rates, suggesting that prenatal diagnosis can prevent the birth of some newborns with CCHD that have no therapeutic value, thereby reducing the financial and emotional burden on families.

The effect of prenatal diagnosis on the survival benefit of CCHD is still unclear. Although Bonnet et al. demonstrated that infants with a prenatal diagnosis of complete transposition of the great artery had lower preoperative and postoperative mortality compared to the postnatal diagnostic group (21), recent studies have failed to confirm this opinion $(28,39,40)$. In another meta-analysis of the impact of prenatal diagnosis of CCHD on surgical outcomes, it was found that the prenatal diagnosis of CCHD improved neonatal survival (18). Our study found that no patients died preoperatively in the prenatal diagnostic group, whereas the number of preoperative deaths in the postnatal diagnostic group was 12 , suggesting that prenatal diagnosis reduces preoperative mortality, which may be related to the fact that prenatal diagnosis allows for better planning of delivery and may improve perinatal and preoperative status. Although we did not find any difference in postoperative mortality and total mortality between the prenatal and postnatal diagnostic groups, we analysed the one-year survival rate of the two groups and found that the one-year survival rate was higher in the prenatal diagnostic group, proving that the integrated management mode of prenatal diagnosispostnatal treatment has a positive effect on the survival of patients with CCHD. 
Neonates born after prenatal diagnosis and prenatal counselling are hospitalized immediately after birth and treated with mechanical ventilation or prostaglandins, depending on their condition, to reduce the occurrence of hypoxia, acidosis and hemodynamic instability, further reducing the occurrence of adverse complications. Calderon et al. (41) reported improved neurocognitive outcomes in infants with a prenatal diagnosis of complete transposition of the great artery compared to their peers with a postnatal diagnosis. Mahle et al. reported higher levels of adverse neurological events in children with hypoplastic left heart syndrome diagnosed only postnatally (27). We found that the incidence of pneumonia and ischemic-ischemic brain damage was higher in the postnatal diagnostic group. Neurocognitive deficits are increasingly recognized as a significant problem in children with CCHD and can significantly affect quality of life for those with the condition. A delayed diagnosis leads to increased risks associated with cardiac dysfunction, brain injury and multiple organ failure.

In summary, through the integrated management mode of prenatal diagnosis-postnatal treatment, CCHD newborns can be closely monitored in utero and promptly undergo active medical and surgical treatment after delivery, thus reducing various postnatal complications, improving the quality of survival and prognosis.

\section{Limitation}

First, this study is limited by the inherent nature of singlecentre retrospective studies. In addition, we could not identify neonates with CCHD who did not have a prenatal diagnosis and died prior to referral to this hospital, so this may reduce the survival advantage of prenatal diagnosis for CCHD. Finally, due to strict inclusion criteria, the number of CCHD cases of some anatomical types included in this study was too small to be analysed separately, which may have interfered with the results and may require a larger study.

\section{Conclusions}

The integrated management mode of prenatal diagnosispostnatal treatment of CCHD allows for better planning of delivery and the possibility of the best medical management of the neonatal period. Prenatal cardiac malformation screening can not only improve the postnatal and surgical status of CCHD newborns, reduce postoperative complications, but also improve the short-term survival rate of patients, which has great social significance. A team of prenatal diagnosis experts should be established to better manage foetal heart disease and help provide better counselling for families.

\section{Acknowledgments}

Funding: This work was supported by National Key R\&D Program of China (2018YFC1002600).

\section{Footnote}

Reporting Checklist: The authors have completed the STROBE reporting checklist. Available at http://dx.doi. org/10.21037/cdt-20-892

Data Sharing Statement: Available at http://dx.doi. org/10.21037/cdt-20-892

Conflicts of Interest: All authors have completed the ICMJE uniform disclosure form (available at http://dx.doi. org/10.21037/cdt-20-892). The authors have no conflicts of interest to declare.

Ethical Statement: The authors are accountable for all aspects of the work in ensuring that questions related to the accuracy or integrity of any part of the work are appropriately investigated and resolved. The study was conducted in accordance with the Declaration of Helsinki (as revised in 2013). The study was approved by the ethics committee of Guangdong Provincial People's Hospital (No. GDREC2018317H) and informed consent was taken from all the patients.

Open Access Statement: This is an Open Access article distributed in accordance with the Creative Commons Attribution-NonCommercial-NoDerivs 4.0 International License (CC BY-NC-ND 4.0), which permits the noncommercial replication and distribution of the article with the strict proviso that no changes or edits are made and the original work is properly cited (including links to both the formal publication through the relevant DOI and the license). See: https://creativecommons.org/licenses/by-nc-nd/4.0/.

\section{References}

1. Dolk H. EUROCAT: 25 years of European surveillance of 
congenital anomalies. Arch Dis Child Fetal Neonatal Ed 2005;90:F355-8.

2. van der Linde D, Konings EEM, Slager MA, et al. Birth prevalence of congenital heart disease worldwide: a systematic review and metaanalysis. J Am Coll Cardiol 2011;58:2241-7.

3. Hoffman JIE. The global burden of congenital heart disease. Cardiovasc J Afr 2013;24:141-5.

4. Abu-Harb M, Hey E, Wren C. Death in infancy from unrecognised congenital heart disease. Arch Dis Child 1994;71:3-7.

5. Schwedler G, Lindinger A, Lange PE, et al. Frequency and spectrum of congenital heart defects among live births in Germany. Clin Res Cardiol 2011;100:1111-7.

6. Germanakis I, Sifakis S. The impact of fetal echocardiography on the prevalence of liveborn congenital heart disease. Pediatr Cardiol 2006;27:465-72.

7. National Health Commission of the People s Republic of China. Report on the prevention and control of birth defects in China. 2012. Available online: http://www.gov. cn/gzdt/att/att/site1/20120912/1c6f6506c7 f811bacf9301. pdf. Accessed 27 June 2018.

8. Fermont L, De Geeter B, Aubry MC, et al. A close collaboration between obstetrician and cardiologists allows antenatal detection of severe cardiac malformations by 2D echocardiography (abstract). Proceedings of the Second World Congress of Paediatric Cardiology. New York 1986:10.

9. Acherman RJ, Evans WN, Luna CF, et al. Prenatal detection of congenital heart disease in southern Nevada: the need for universal fetal cardiac evaluation. J Ultrasound Med 2007;26:1715-9; quiz 1720-21.

10. Chu C, Yan Y, Ren Y, et al. Prenatal diagnosis of congenital heart diseases by fetal echocardiography in second trimester: a Chinese multicenter study. Acta Obstet Gynecol Scand 2017;96:454-63.

11. Landis BJ, Levey A, Levasseur SM, et al. Prenatal diagnosis of congenital heart disease and birth outcomes. Pediatr Cardiol 2013;34:597-605.

12. Garne E, Stoll C, Clementi M. Evaluation of prenatal diagnosis of congenital heart diseases by ultrasound: experience from 20 European registries. Ultrasound Obstet Gynecol 2001;17:386-91.

13. Jørgensen DE, Vejlstrup N, Jorgensen C, et al. Prenatal detection of congenital heart disease in a low risk population undergoing first and second trimester screening. Prenat Diagn 2015;35:325-30.

14. Friedberg MK, Silverman NH, Moon-Grady AJ, et al.
Prenatal detection of congenital heart disease. J Pediatr 2009;155:26-31, 31.e21.

15. Bortnick AE. Support of the failing left ventricle: extracorporeal life support plus blade and balloon atrioseptostomy as an alternative option. J Interv Cardiol 2012;25:68-70.

16. Dahdouh Z, Roule V, Sabatier R, et al. Extra-corporeal life support, transradial thrombus aspiration and stenting, percutaneous blade and balloon atrioseptostomy, all as a bridge to heart transplantation to save one life. Cardiovasc Revasc Med 2012;13:241-5.

17. Oster ME, Kim CH, Kusano AS, et al. A PopulationBased Study of the Association of Prenatal Diagnosis With Survival Rate for Infants With Congenital Heart Defects. Am J Cardiol 2014;113:1036-40.

18. Holland BJ, Myers JA, Woods CR Jr. Prenatal diagnosis of critical congenital heart disease reduces risk of death from cardiovascular compromise prior to planned neonatal cardiac surgery: a meta-analysis. Ultrasound Obstet Gynecol 2015;45:631-8.

19. Franklin O, Burch M, Manning N, et al. Prenatal diagnosis of coarctation of the aorta improves survival and reduces morbidity. Heart 2002;87:67-9.

20. Verheijen PM, Lisowski LA, Stoutenbeek P, et al. Lactacidosis in the neonate is minimized by prenatal detection of congenital heart disease. Ultrasound Obstet Gynecol 2002;19:552-5.

21. Bonnet D, Coltri A, Butera G, et al. Detection of transposition of the great arteries in fetuses reduces neonatal morbidity and mortality. Circulation 1999;99:916-8.

22. Eapen RS, Rowland DG, Franklin WH. Effect of prenatal diagnosis of critical left heart obstruction on perinatal morbidity and mortality. Am J Perinatol 1998;15:237-42.

23. Satomi G, Yasukochi S, Shimizu T, et al. Has fetal echocardiography improved the prognosis of congenital heart disease? Comparison of patients with hypoplastic left heart syndrome with and without prenatal diagnosis. Pediatr Int 1999;41:728-32.

24. Verheijen PM, Lisowski LA, Stoutenbeek P, et al. Prenatal diagnosis of congenital heart disease affects preoperative acidosis in the newborn patient. J Thorac Cardiovasc Surg 2001;121:798-803.

25. Kipps AK, Feuille C, Azakie A, et al. Prenatal diagnosis of hypoplastic left heart syndrome in current era. Am J Cardiol 2011;108:421-7.

26. Kumar RK, Newburger JW, Gauvreau K, et al. Comparison of outcome when hypoplastic left heart 
syndrome and transposition of the great arteries are diagnosed prenatally versus when diagnosis of these two conditions is made only postnatally. Am J Cardiol 1999;83:1649-53.

27. Mahle WT, Clancy RR, McGaurn SP, et al. Impact of prenatal diagnosis on survival and early neurologic morbidity in neonates with the hypoplastic left heart syndrome. Pediatrics 2001;107:1277-82.

28. Levey A, Glickstein JS, Kleinman CS, et al. The impact of prenatal diagnosis of complex congenital heart disease on neonatal outcomes. Pediatr Cardiol 2010;31:587-97.

29. Munn MB, Brumfield CG, Lau Y, et al. Prenatally diagnosed hypoplastic left heart syndrome outcomes after postnatal surgery. J Matern Fetal Med 1999;8:147-50.

30. Tuo G, Volpe P, Bondanza S, et al. Impact of prenatal diagnosis on outcome of pulmonary atresia and intact ventricular septum. J Matern Fetal Neonatal Med 2012;25:669-74.

31. Hancock HS, Romano JC, Armstrong A, et al. Single Ventricle and Total Anomalous Pulmonary Venous Connection: Implications of Prenatal Diagnosis. World J Pediatr Congenit Heart Surg 2018;9:434-9.

32. Plana MN, Zamora J, Suresh G, et al. Pulse oximetry screening for critical congenital heart defects. Cochrane Database Syst Rev 2018;(3):CD011912.

33. Kemper AR, Mahle WT, Martin GR, et al. Strategies for implementing screening for critical congenital heart disease. Pediatrics 2011;128:e1259-67.

34. Mahle WT, Newburger JW, Matherne GP, et al. Role

Cite this article as: Zhang X, He S, Liu Y, Zhong J, Sun Y, Zheng M, Gui J, Wang R, Feng B, Mo J, Jian M, Liu C, Liang Y. The significance of an integrated management mode of prenatal diagnosis-postnatal treatment for critical congenital heart disease in newborns. Cardiovasc Diagn Ther 2021;11(2):447456. doi: $10.21037 / \mathrm{cdt}-20-892$ of pulse oximetry in examining newborns for congenital heart disease: a scientific statement from the American Heart Association and American Academy of Pediatrics. Circulation 2009;120:447-58.

35. Ferencz C, Rubin JD, McCarter RJ, et al. Congenital heart disease: prevalence at livebirth. The BaltimoreWashington Infant Study. Am J Epidemiol 1985;121:31-6.

36. Rosano A, Botto LD, Botting B, et al. Infant mortality and congenital anomalies from 1950 to 1994: an international perspective. J Epidemiol Community Health 2000;54:660-6.

37. Raboisson MJ, Samson C, Ducreux C, et al. Impact of prenatal diagnosis of transposition of the great arteries on obstetric and early postnatal management. Eur J Obstet Gynecol Reprod Biol 2009;142:18-22.

38. Wright LK, Ehrlich A, Stauffer N, et al. Relation of prenatal diagnosis with one-year survival rate for infants with congenital heart disease. Am J Cardiol 2014;113:1041-4.

39. Yates RS. The influence of prenatal diagnosis on postnatal outcome in patients with structural congenital heart disease. Prenat Diagn 2004;24:1143-9.

40. Sivarajan V, Penny DJ, Filan P, et al. Impact of antenatal diagnosis of hypoplastic left heart syndrome on the clinical presentation and surgical outcomes: the Australian experience. J Paediatr Child Health 2009;45:112-7.

41. Calderon J, Angeard N, Moutier S, et al. Impact of prenatal diagnosis on neurocognitive outcomes in children with transposition of the great arteries. J Pediatr 2012;161:94-98.e91. 